\title{
Análise da relação entre espessura macular e espessura da camada de fibras nervosas
}

\author{
Analysis of the relation between macular thickness \\ and thickness of the fiber nervous layers
}

Fernando Costa Boucault ${ }^{1}$, Rodrigo Thompson de Moura², Jorge Mitre ${ }^{3}$, José Ricardo Carvalho Lima Rehder ${ }^{4}$

\begin{tabular}{|l|}
\hline \multicolumn{1}{|c|}{ RESUMO } \\
Objetivo: O objetivo desse trabalho é avaliar por meio do OCT Stratus a relação da \\
espessura macular com a espessura de camadas de fibras nervosas. Métodos: Neste \\
trabalho foram analisados 106 olhos do serviço de oftalmologia da Faculdade de Medi- \\
cina do ABC. Estudou-se 55 olhos que apresentavam valores de CFNR dentro ou acima \\
dos valores normais em todos o quatro quadrantes e 51 olhos apresentando valores em \\
apenas um ou mais quadrantes que indique suspeita ou fora do limite de normalidade \\
dos valores de CFNR, comparando com sua espessura macular. Resultados: Este estudo \\
mostrou uma diminuição da espessura macular nos pacientes que apresentavam sus- \\
peitas ou diminuição da espessura de CFNR. Conclusão: O trabalho demonstrou que a \\
espessura macular pode ser importante para avaliar uma possível perda de camada de \\
fibras nervosas.
\end{tabular}

Descritores: Mácula lútea/fisiologia; Fibras nervosas;Tomografia de coerência óptica/ métodos; Valores de referência; Retina

\footnotetext{
${ }^{1}$ Médico Voluntário do Setor de Retina e Vítreo do Departamento de Oftalmologia da Faculdade de Medicina do ABC - FMABC Santo André (SP), Brasil;

${ }^{2}$ Médico Voluntário do Setor de Retina e Vítreo do Departamento de Oftalmologia da Faculdade de Medicina do ABC - FMABC Santo André (SP), Brasil;

${ }^{3}$ Chefe do Setor de Retina e Vítreo do Departamento de Oftalmologia da Faculdade de Medicina do ABC - FMABC - Santo André (SP), Brasil;

${ }^{4}$ Professor Titular do Departamento de Oftalmologia da Faculdade de Medicina do ABC - FMABC - Santo André (SP), Brasil;

Trabalho realizado no Departamento de Oftalmologia da Faculdade de Medicina do ABC - FMABC - Santo André (SP), Brasil.

Recebido para publicação em: 18/9/2009 - Aceito para publicação em 22/8/2010
} 


\section{INTRODUÇÃO}

$\mathbf{0}$ tomógrafo de coerência óptica, OCT Stratus (Carl Zeiss Ophthalmic Systems, Inc. EUA) é um moderno equipamento que permite a realização de cortes transversais da retina, gerando imagens tomográficas de alta resolução, com alto grau de reprodutibilidade ${ }^{(1-2)}$. É especialmente útil para aplicações diagnósticas oftalmológicas, devido ao fácil acesso óptico às estruturas do segmento posterior do olho. A imagem produzida é resultante de um corte transversal tecidual, com resolução que se aproxima à microscopia de luz, sem requerer biópsia cirúrgica, que denominamos de "biópsia óptica". O OCT (do inglês optical coherence tomography) possui um mecanismo análogo ao ultrassom no modo B, utilizando luz com comprimento de onda infravermelho ao invés da onda sonora ${ }^{(3)}$. Nessa técnica, a luz é altamente refletida ou absorvida pelos tecidos biológicos, o que a torna adequada para avaliar estruturas intraoculares opticamente acessíveis.

É um exame de alta resolução $(10 \mu \mathrm{m})$, que realiza cortes seccionais da retina e coróide, capaz de detectar alterações anatômicas coroido-retinianas no plano z (profundidade da retina). As alterações detectadas são observadas como alterações relativas de refletividade na interface óptica obtidas pelo uso do método de interferometria de baixa coerência ${ }^{(4)}$.

O uso dessa nova forma de estudo pode observar melhor a interação de doenças com o padrão retiniano, fazendo correlações das diferentes formas apresentadas, como no caso das Camadas de Fibra Nervosas Retinianas (CFNR), meio de estudo utilizado para a evolução do glaucoma com a Espessura Macular (EM), correlacionando ambos para demonstrar se existe uma nova arma para o diagnóstico precoce das doenças que evoluem com a perda da CFNR.

Apesar da grande maioria dos estudos terem avaliado o dano neural através da análise da CFNR peridiscal, trabalhos recentes demonstraram que a medida da espessura macular também pode ser importante para estimar o dano neural ${ }^{(5-6)}$.

O objetivo desse estudo é correlacionar a perda da camada de fibras nervosas da retina com a diminuição na espessura macular.

\section{Métodos}

Foram analisados 106 olhos do serviço de oftalmologia da Faculdade de Medicina do ABC, onde não portavam doenças que pudessem gerar alguma alteração

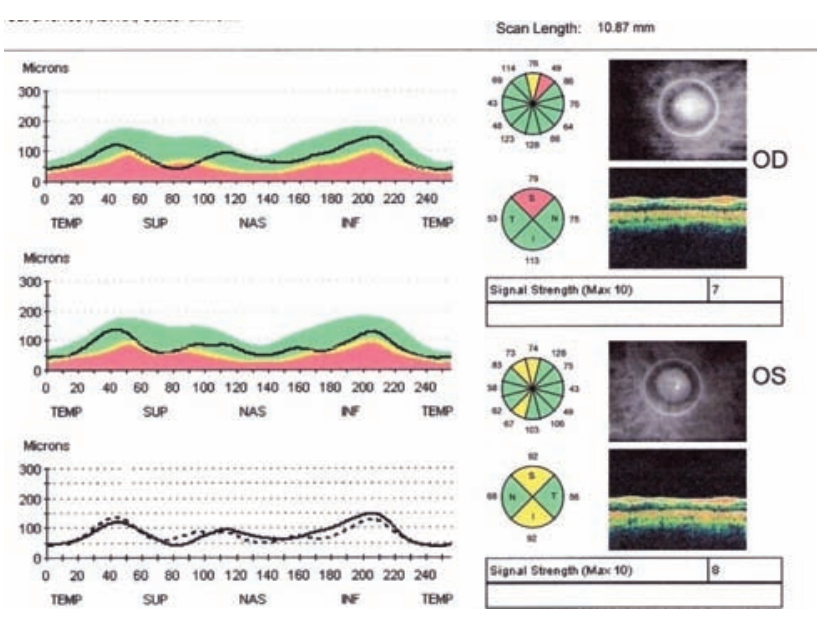

Figura 1: Protocolo RNFL Thickness Average utilizado no OCT Stratus

macular, como: Diabetes, sequela de oclusão vascular, buraco macular, degeneração macular relacionada à idade, bem como outras patologias. Utilizamos o protocolo RNFL Thickness Average que é aplicado sobre uma determinada imagem, aparece à imagem normativa com os dados corrigidos para a faixa etária. O programa utiliza as cores branco (acima dos valores considerados normais, $>95 \%$ ), verde (dentro dos valores normais), amarela (indicam suspeita, $<5 \%$ ) e vermelha (fora do limite de normalidade, $<1 \%$ ) para indicar os percentis de distribuição normal (Figura 1). Todo o banco de dados está baseado em medidas circulares de 3,4 mm de diâmetro posicionados ao redor do disco óptico ${ }^{(3)}$.

Foram estudados 55 olhos que apresentavam valores de CFNR dentro ou acima dos valores normais em todos o quatro quadrantes e 51 olhos apresentando valores em apenas um ou mais quadrantes que indique suspeita ou fora do limite de normalidade dos valores de CFNR, comparando com sua espessura macular utilizando Macular Thikness Map, que consiste em uma série de 6 cortes igualmente espaçados, com um ponto central em comum, possuindo cortes de tamanho fixo de $6,0 \mathrm{~mm}$, sendo utilizado no exame de espessura macular. O protocolo de análise desses cortes foi o Retinal Map, que oferece dois mapas da espessura retiniana em uma área circular centrada na mácula. Um mapa mostra a espessura retiniana usando um código de cor, e o outro mostra espessura retiniana média (em micra) de nove setores do mapa, utilizamos nesse estudo como dado o setor numérico central, por se tratar da área foveolar do corte.

Os pacientes tinham idade acima de 50 anos com média de 66,5 anos, sendo $34 \%$ do sexo masculino (Gráfico 1). Foram utilizados colírios para dilatação para a 

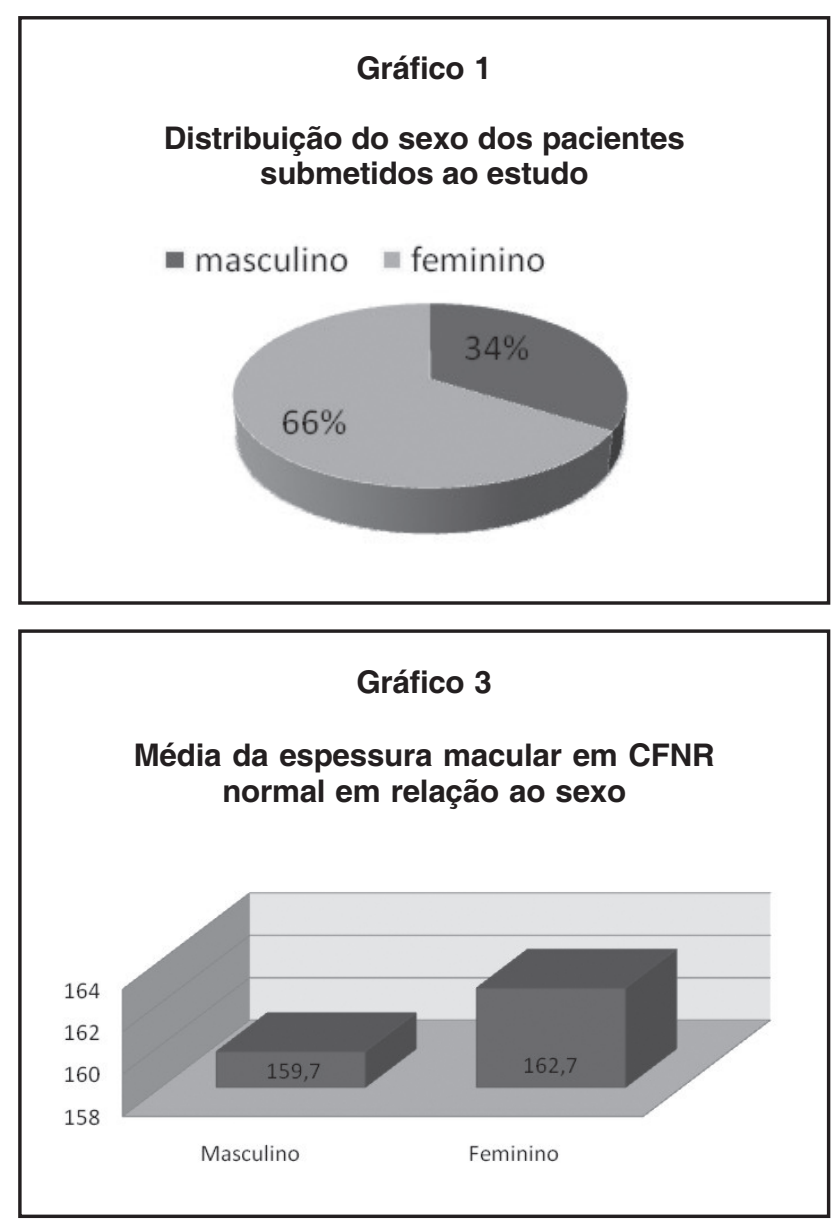

obtenção da imagem, não tendo assim interferência a área pupilar, sendo também realizado pelo mesmo examinador em todos os casos.

\section{Resultados}

O estudo mostrou uma diminuição da espessura macular nos pacientes que apresentavam suspeitas ou diminuição da espessura de CFNR em relação aos pacientes com espessura dentro ou acima da normalidade.

Os valores médios foram de 161,2 $\mu \mathrm{m}$ (117-214) para pacientes dentro ou acima da normalidade versus $154,4 \mu \mathrm{m}(85-193)$ para pacientes suspeitos ou inferiores ao padrão de normalidade, sendo a diferença de 6,8 $\mu \mathrm{m}$ para os valores acima (Gráfico 2).

Pacientes do sexo masculino apresentaram média menor na espessura macular, $150,9 \mu \mathrm{m}$ em relação ao sexo feminino, $157,9 \mu \mathrm{m}$ nos suspeitos e inferiores aos padrões de normalidades (Gráfico 3), já nos pacientes dentro ou acima da normalidade a diferença foi de $159,7 \mu \mathrm{m}$ contra $162,7 \mu \mathrm{m}$ do sexo masculino e feminino respectivamente (Gráfico 4).
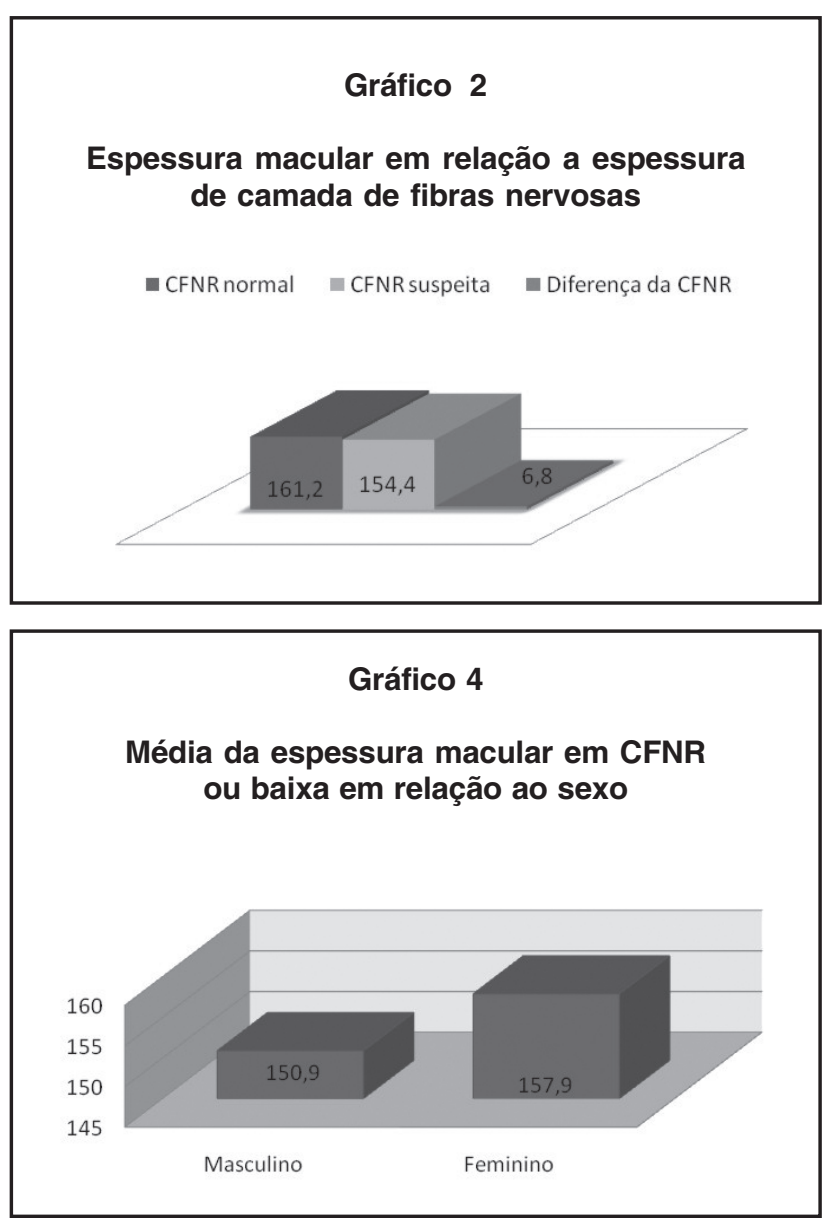

\section{DıscussÃo}

Este estudo revelou que existe uma significativa diferença entre a espessura macular em pacientes normais em relação à espessura de CFNR, aos de menores espessuras de CFNR.

Alguns estudos demonstraram que a perda neural na mácula pode se apresentar sob diferentes formas, podendo apresentar um padrão de perda difusa no glaucoma e na neuropatia óptica traumática ou hemianópico na atrofia em banda do nervo óptico ${ }^{(5-7)}$.

A espessura macular em pacientes normais não é uniforme nas diferentes versões do OCT ${ }^{(8-9)}$. Recentemente, Chan et al. ${ }^{(9)}$ reportaram que, utilizando Stratus OCT, a espessura foveal central em pacientes normais variava em média de $182 \pm 23 \mu \mathrm{m}$, quando determinada automaticamente pelo aparelho e de $170 \pm$ $18 \mu \mathrm{m}$, quando determinada manualmente.

A camada de células ganglionares da retina correspondem a cerca de 35 a $40 \%$ da espessura retiniana na área macular. Sendo assim, estimativas da 
espessura macular podem ser consideradas como uma forma de avaliarmos a perda de células ganglionares em afecções da via óptica anterior ${ }^{(5-10)}$. Alguns estudos demonstraram que a espessura macular medida pelo OCT em pacientes glaucomatosos é significativamente menor quando comparada a de indivíduos normais ${ }^{(5-6)}$.

Devemos, no entanto, atentar para o fato de que outras afecções da mácula podem influenciar na avaliação da perda neural quando esta é estimada pela espessura macular. Desta forma, afecções que cursam com edema macular, membranas epi ou sub-retinianas, cicatrizes ou degenerações maculares podem causar alterações na espessura macular. Desta forma, enquanto que a quantificação da perda neural feita pela medida da CFNR sofre influência da presença de edema ou anomalias de disco óptico, a avaliação feita pela espessura macular também pode sofrer influência de outras afecções maculares, particularmente em indivíduos idosos e diabéticos nos quais devemos ter cuidado ainda maior na interpretação dos achados. Devemos também lembrar que tais achados devem ser correlacionados com os dados clínicos, particularmente os exames da função visual como o campo visual. Salientamos, por fim, que a tomografia de coerência óptica não deve ser solicitada de forma indiscriminada e sim como um complemento na investigação de casos nos quais existam dúvidas diagnósticas ou necessidade de quantificação e seguimento do dano axonal retiniano.

\section{Conclusão}

O trabalho demonstrou que a espessura macular é importante para avaliar uma possível perda de camada de fibras nervosas, o que é muito importante para o diagnóstico precoce de doenças como glaucoma e neuropatias. Salientamos que o OCT é um exame subsidiário não podendo ser trocado pela avaliação clínica, tendo então sua indicação restrita para casos selecionados.

\section{Abstract}

Objective: The objective of this work is to evaluate by means of the OCT Stratus the relation of the macular thickness with the thickness ofnervous fiber layers. Methods: In this work 106 eyes of the service of ophthalmology of the College of Medicine of the ABC had been analyzed. The study included 55 eyes that inside presented values of CFNR or above of the normal values in all the four quadrants and 51 eyes presenting values in only one or more quadrants that suspicion indicates or is of the limit of normality of the values of CFNR, comparing with macular thickness. Results: This study it showed a reduction of the macular thickness in the patients who presented suspicion or reduction of the thickness of CFNR. Conclusion: The work demonstrated that the macular thickness can be a weapon important to evaluate a possible loss of nervous fiber layer.

Keywords: Macula lutea/physiology; Nerve fibers; Tomography, optical coherence/methods; Reference values; Retina

\section{RefERÊNCIAS}

1. Hee MR, Izatt JA, Swanson EA, Huang D, Schuman JS, Lin $\mathrm{CP}$, et al. Optical coherence tomography of the human retina. Arch Ophthalmol.1995;113(3):325-32.

2. Puliafito CA, Hee MR, Schuman JS, Fujimoto JG. Principles of operation and technology. Thorofare (New Jersey): Slack; 1995. Optical coherence tomografy of ocular diseases; p. 3-15.

3. Farah ME. Tomografia de coerência óptica- OCT- Texto e Atlas. Rio de Janeiro: Cultura Médica; 2006.

4. Mesquita JR. Técnicas de exame. Apresentação do StratusOCT. In: XXIX Congresso da Sociedade Brasileiro de Retina e Vítreo; 2004; Florianópolis.

5. Guedes V, Schuman JS, Hertzmark E, Wollstein G, Correnti A, Mancini R, et al. Optical coherence tomography measurement of macular and nerve fiber layer thickness in normal and glaucomatous human eyes. Ophthalmology. 2003;110 (1):177-89.

6. Moura FC, Medeiros FA, Monteiro ML. Evaluation of macular thickness measurements for detection of band atrophy of the optic nerve using optical coherence tomography. Ophthalmology. 2007;114(1):175-81.

7. Vessani RM, Cunha LP, Monteiro ML. Progressive macular thinning after indirect traumatic optic neuropathy documented by optical coherence tomography. $\mathrm{Br} \mathrm{J}$ Ophthalmol. 2007;91(5):697-8.

8. Chan A, Duker JS, Ko TH, Fujimoto JG, Schuman JS. Normal macular thickness measurements in healthy eyes using Stratus optical coherence tomography. Arch Ophthalmol. 2006;124(2):193-8.

9. Paunescu LA, Schuman JS, Price LL, Stark PC, Beaton S, Ishikawa $\mathrm{H}$, et al. Reproducibility of nerve fiber thickness, macular thickness, and optic nerve head measurements using StratusOCT. Invest Ophthalmol Vis Sci. 2004; 45(6):1716-24.

10. Cunha LP, Vessani RM, Monteiro MLR. Detecção de perda neural localizada através da redução da espessura macular na tomografia de coerência óptica: relato de caso. Arq Bras Oftalmol. 2008; 71(5):743-6.

\author{
Endereço para correspondência: \\ Fernando Costa Boucault \\ Avenida Laurinda Cardoso Mello Freire, no 396 - Vila \\ Oliveira \\ CEP 08780-280 - Mogi das Cruzes (SP), Brasil \\ Tel: (11) 4799-5540 \\ e-mail: drfernando@oftalmoboucault.com.br
}

